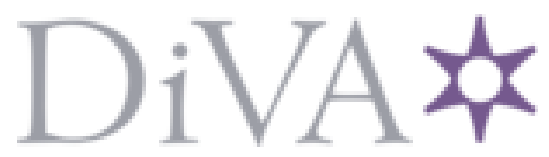

http://www.diva-portal.org

This is the published version of a paper published in Journal of Novel Physiotherapies.

Citation for the original published paper (version of record):

Nyberg, A., Lindström, B., Wadell, K. (2014)

A Cohort Study to Evaluate the Feasibility of Low Load/High Repetition Elastic Band

Resistance Training for People with Chronic Obstructive Pulmonary Disease.

Journal of Novel Physiotherapies, 4

http://dx.doi.org/10.4172/2165-7025.1000190

Access to the published version may require subscription.

N.B. When citing this work, cite the original published paper.

Permanent link to this version:

http://urn.kb.se/resolve?urn=urn:nbn:se:umu:diva-87859 


\title{
A Cohort Study to Evaluate the Feasibility of Low Load/High Repetition Elastic Band Resistance Training for People with Chronic Obstructive Pulmonary Disease
}

\author{
Andre Nyberg ${ }^{1 *}$, Britta Lindstrom ${ }^{1}$ and Karin Wadell ${ }^{1,2}$ \\ ${ }^{1}$ Department of Community Medicine and Rehabilitation, Physiotherapy, Umea University, Umea, Sweden \\ ${ }^{2}$ Department of Public Health and Clinical Medicine, Division of Medicine, Umea University, Umea, Sweden
}

\begin{abstract}
Background: Resistance training is an important component of pulmonary rehabilitation in people with Chronic Obstructive Pulmonary Disease (COPD). A vast majority or resistance training studies in COPD have focused on increasing muscular strength with use of weight machines and has been found to be a feasible approach in COPD. However, regarding feasibility of resistance training equipment other than weight machines, such as elastic resistance equipment, information is scarce. In addition, little is known regarding other trainable muscle characteristics, such as peripheral muscular endurance in COPD. We therefore aim to evaluate the feasibility of a low load/high repetition elastic band Resistance Training (RT) regimen in people with moderate to severe COPD. We also aim to evaluate if the RT regimen is feasible for the physiotherapists, conducting the intervention.
\end{abstract}

Methods: Twenty-two participants with moderate to severe COPD (mean forced expiratory volume in one second, FEV $158.7 \%$ predicted) and five physiotherapists participated in the study. Measurements of attendance, assessment and progression of exercise intensity, adverse events, participant and compliance (participant and physiotherapist) were collected for assessment of feasibility.

Results: The mean (95\% confidence interval) attendance rate was $94 \%(91-97)$ and mean exercise intensity was $79 \%$ (74-83) of the predicted maximum intensity. Furthermore, the median (interquartile range) compliance was $96 \%$ (94-96) and 100\% (94-100) for the people with COPD and the physiotherapists respectively. Exercise intensity increased on all exercises and any recorded adverse events were considered minor and temporary.

Conclusions: Low load/high repetition elastic band resistance training appears to be feasible for both people with COPD and the physiotherapists performing the intervention. These results indicate that elastic bands could be a feasible alternative to weight machines in resistance training for people with COPD.

Keywords: Pulmonary disease; Chronic obstructive; Feasibility; Resistance training; Peripheral muscular endurance; Single limb; Elastic resistance

\section{Introduction}

Pulmonary rehabilitation is effective for people with Chronic Obstructive Pulmonary Disease (COPD) with regard to symptoms, function, participation, and health-care resource utilization [1]. One of the components of this evidence-based comprehensive intervention is resistance training that has been found to be beneficial in improving muscular strength, cycle endurance capacity [2-4] and upper extremity functional capacity [4]. In COPD, the predominant choice of exercise equipment in resistance training regimens has been weight machines. However, lack of access to this exercise equipment can be a barrier to maintenance of exercise capacity. This has highlighted the need to examine effects and safety of alternate exercise equipment [3]. Elastic resistance equipment such as elastic bands and tubing offers an alternative that has shown comparable results to weight machines regarding both upper and lower extremity exercises in healthy adults [5,6]. In people with COPD, O'Shea et al. [7] found that a predominantly home-based resistance training regimen with elastic bands could be used to increase knee extensor strength. Nevertheless, they did not find any effect on upper extremity strength and walking ability. The authors argued that the high attrition rate, with more than $40 \%$ of participants unable to complete the exercise program, to some extent may have influenced the effectiveness of the exercise regimen. This is, to our knowledge the only published study that have used elastic bands as primary resistance in people with COPD. In addition the study did not report any information about feasibility of the exercise program such as adverse events and compliance. Therefore, knowledge concerning the feasibility of using resistance training with elastic bands as resistance is limited for this group of people.

A majority of previous resistance training studies in COPD have primarily focused on increasing muscular strength. Little is known regarding other trainable characteristics, such as peripheral muscular endurance which may be of importance since quadriceps muscular endurance has been shown to be reduced to a larger extent in comparison to quadriceps strength in people with COPD [8].

We have recently submitted a paper on the effects of an eight week low load/high repetition (i.e. peripheral muscular endurance) elastic band resistance training regimen that showed promising results, including increased functional upper and lower extremity exercise capacity, maximal strength and peripheral muscular endurance for the

*Corresponding author: Andre Nyberg, Department of Community Medicine and Rehabilitation, Physiotherapy, Umea University, 90187 Umea, Sweden, Tel: + 46 90786 9089; Fax: + 4690786 9267; E-mail: andre.nyberg@physiother.umu.se

Received December 05, 2013; Accepted January 07, 2014; Published January 09, 2014

Citation: Nyberg A, Lindstrom B, Wadell K (2014) A Cohort Study to Evaluate the Feasibility of Low Load/High Repetition Elastic Band Resistance Training for People with Chronic Obstructive Pulmonary Disease. J Nov Physiother 4: 190 doi:10.4172/2165-7025.1000190

Copyright: ( 2014 Nyberg A, et al. This is an open-access article distributed under the terms of the Creative Commons Attribution License, which permits unrestricted use, distribution, and reproduction in any medium, provided the original author and source are credited. 
people with COPD. However the feasibility of this exercise regimen in people with COPD is still unknown.

Therefore, the aim of the present study was to investigate if:

1. A low load/high repetition elastic band Resistance Training (RT) regimen is feasible in people with COPD?

2. The RT regimen is considered feasible for the physiotherapists supervising and conducting the intervention in clinical settings?

\section{Method}

\section{Design}

The study was performed on people with COPD who were randomly assigned to participate in an eight week RT regimen [9]. The present study was approved by the Regional Ethical Board, Umeå University, Umeå, Sweden (Dnr: 2010-344-31 M) and was registered at www.clinicaltrials.gov (NCT01354067). The study is reported in line with strengthening the reporting of observational studies in epidemiology guideline [10]. Recruitment, data collection and followup were performed from August 15, 2011 to May 3, 2012 [9].

Eligibility criteria for patients included having stable COPD stage II-IV according to the Global Initiative for Chronic Obstructive Lung Disease guidelines [11], ex-smoker, on stable medication and randomized to participate in the RT regimen. Eligibility criteria for physiotherapists included at least two years' experience of working as a physiotherapist, one year experience of working with cardiorespiratory patients, and at least one year experience of leading training groups [9]. Written informed consent was obtained from both patients and physiotherapists.

\section{Interventions}

The RT regimen is a standardized resistance training regimen which consists of eight upper and lower extremity exercises performed with one leg/arm at a time. Six exercises are performed with elastic bands (Thera-Bands ${ }^{\circledR}$, The Hygenic Corporation) and two with body weight (heel-raise and step-up) as primary resistance (Figure 1).

The RT regimen was conducted in a group format and supervised by physiotherapists. Each group consisted of three to seven patients who attended three sessions per week during eight weeks. The sessions lasted approximately 60 minutes including warm-up, exercise and cooldown, each part designed in accordance to American College of Sports Medicine recommendations [12-14]. Volume, initial load, rest periods, frequency, repetition velocity, and progression in the RT regimen were standardized in accordance with recommendations for increasing peripheral muscular endurance $[9,14]$. The exercises within the RT regimen target muscles with decreased strength/endurance (compared to healthy individuals), muscles which are important for upper extremity activities of daily living, and muscles which are important for walking [15-18]. All exercises were executed using a single limb at a time, alternating left to right side in order to minimize the risk of a central constraint [19]. Execution of all exercise was standardized to optimize activation of targeted muscles, and included execution in the most clinically effective portion of elastic resistance [2,9,20-22]. The length of each individual elastic band, patient position from elastic resistance insertion and, start and stop positions for each exercise, were also standardized [9,23]. All components were individually determined and progressed with use of Borg category ratio (CR10) scale [24], according to a specified protocol to optimize individual tailoring of the exercise regimen [9]. The physiotherapists were given a standardized education session in order to deliver a uniform intervention and the exercise protocol was given to both people with COPD and physiotherapists to enhance adherence and familiarization. Written commitments to follow to the assigned protocol were signed by all physiotherapists. Further description on the components and standardization of the exercise regimen are available [9].

\section{Outcomes and follow-up}

Feasibility of the RT regimen was assessed by the attendance rate,

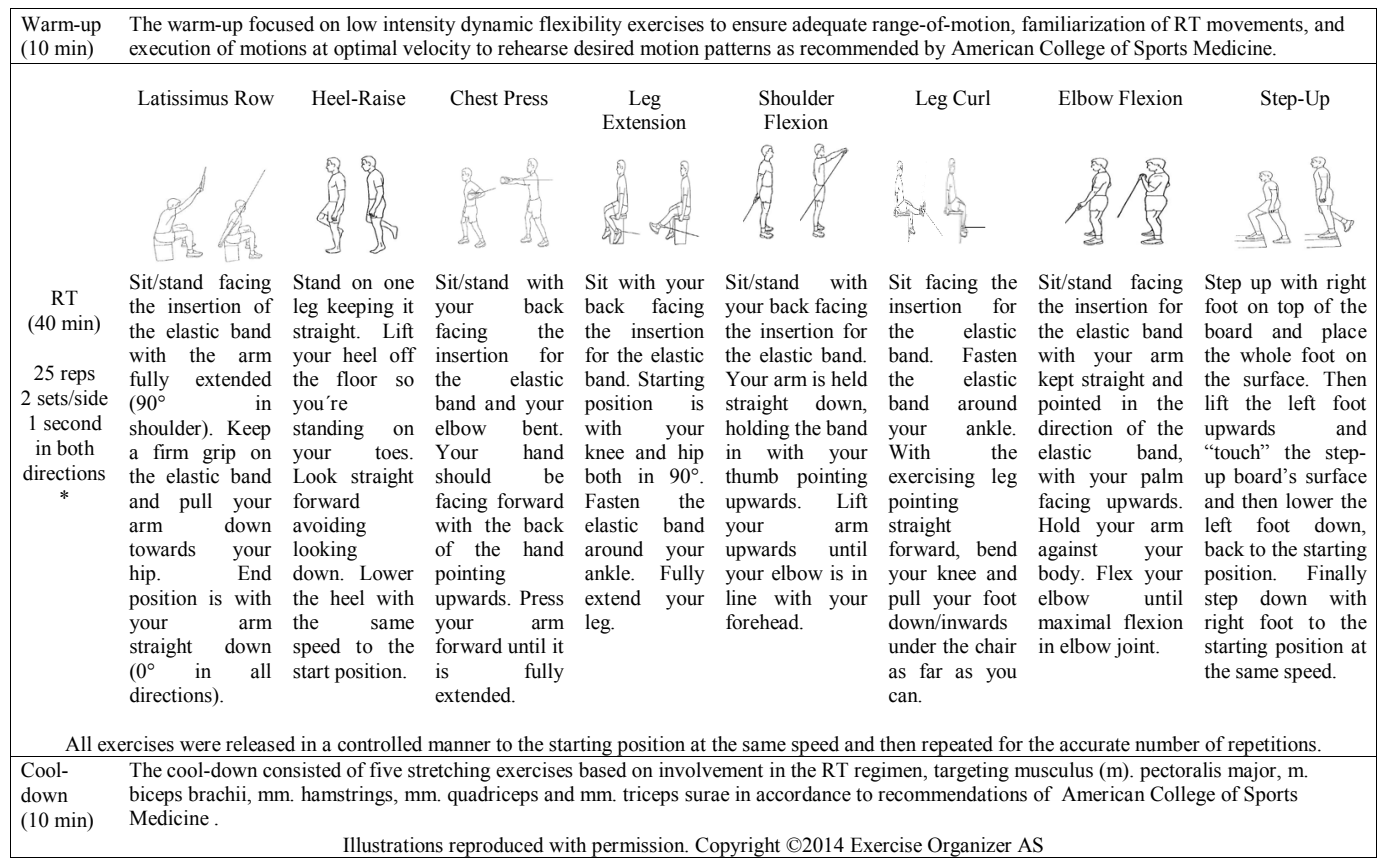

Figure 1: Description of the exercise regimen. $\mathrm{RT}=$ low load/high repetition elastic band resistance training *the step-up exercise was performed using the patients self-paced speed. 
exercise intensity, compliance and occurrence and severity of any adverse events. All outcome data was collected between the baseline tests and within three weeks after the end of the eight-week intervention.

Attendance was evaluated by calculating an attendance rate for each patient as the number of attended sessions divided by total number of sessions.

Exercise intensity was evaluated through assessment and progression of the intensity. Mean muscle fatigue rating on the Borg CR10 scale during the whole intervention period was divided by muscle fatigue rating at a baseline 25 repetition maximum test to determine exercise intensity. The ratings during exercise training were done independently by the patients by pointing to a Borg CR10 scale for dyspnea and muscle fatigue, noted by the physiotherapist. Progression of exercise intensity was evaluated by comparing exercise intensity between the first and last exercise session.

Information about adverse events was collected from exercise diaries and post training interviews with the physiotherapists who led the interventions. Two independent pulmonologists and one physiotherapist who were not involved in the study evaluated the adverse events. The severity of them was assessed and rated into four different categories: (1) minor and temporary, (2) serious symptoms (potential risk of severe injury or life threatening), (3) manifest injury or disease, and (4) death as previously used [25]. An adverse event rate was calculated for each patient as the total number of sessions in which any adverse events occurred divided by the total number of attended sessions.

Compliance was evaluated by a standardized questionnaire. The questions, ranging from 1 to 5 , focused on the extent to which they could follow the instructions on the different components of the intervention. A rating of 1-3 was defined as a small extent and 4-5 large extent. The dichotomization was used to simplify the understanding of the interpretation of compliance to the exercise regimen.

\section{Statistical analysis}

Data analyses included all patients using intention-to-treat analysis. Data was missing on the compliance questionnaire from one participant with COPD due to an exacerbation after the end-ofintervention period. This missing data was considered Missing Not At Random (MNAR). Missing ratings was due to the physiotherapists forgetting to note the rating, which was considered Missing At Random (MAR) [26]. MNAR and MAR data was imputed using the overall mean [27]. A sensitivity analysis was performed comparing the results with imputed data to a complete case analysis [10]. Comparison between participants and physiotherapists regarding compliance were performed using the Chi-square test. Paired sample t-test and Wilcoxon signed rank test was used for exercise intensity and complete-case comparisons when appropriate. Data is presented in mean $(95 \%$ confidence interval $(95 \% \mathrm{CI})$ ) or median (interquartile range (IQR)) depending on distribution of data unless otherwise stated. No additional analyses were made. A p value of $<.05$ was considered statistically significant. For data management and statistical analysis the IBM Statistical Package for Social Sciences (SPSS) version 20.0 was used. Additional details of the methods used are provided in study protocol [9].

\section{Results}

The RT regimen was implemented and administered according to trial protocol [9]. All participants completed the intervention (Figure 2). All data was collected within three weeks after completing the intervention. Participant characteristics are presented in Table 1 and experience of the five physiotherapists is presented in Table 2 .

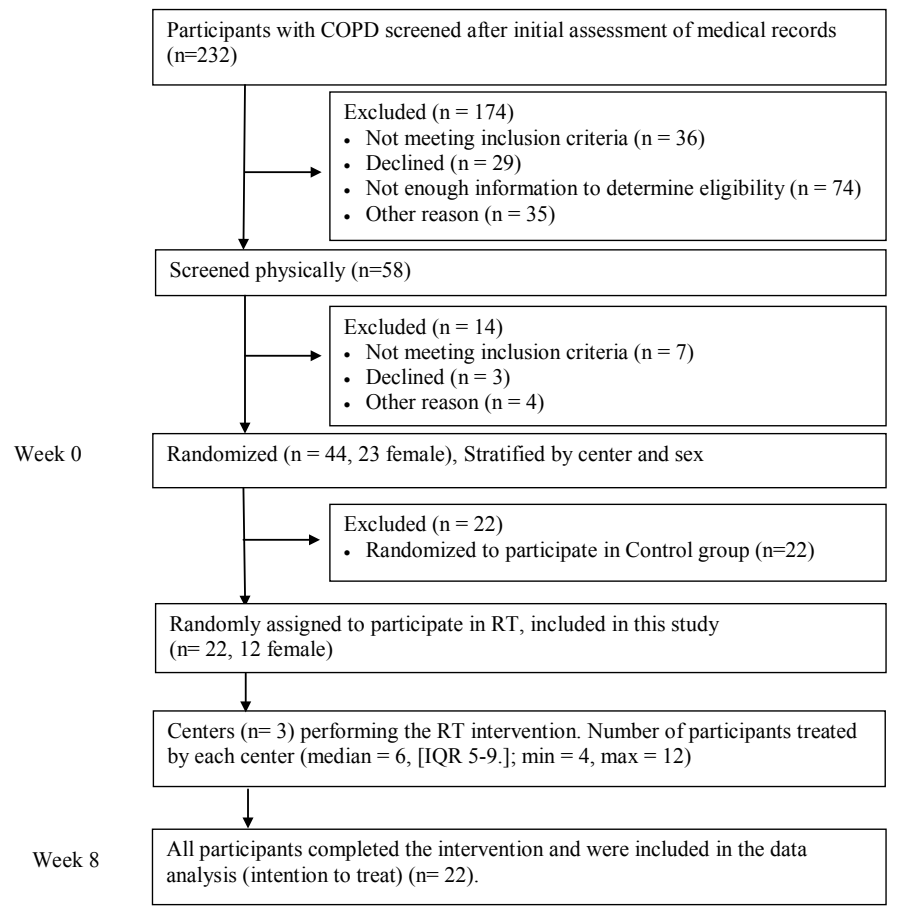

Figure 2: Flow diagram of participants through the different stages of the study. IQR=Interquartile Range, Max=Maximum; Min=Minimum, RT=Low Load/High Repetition Elastic Band Resistance Training. 


\begin{tabular}{|l|l|}
\hline Participants & \\
\hline Age (years) & $69(5)$ \\
\hline Sex (female) & $12(55 \%)$ \\
\hline Body mass index (BMI) & $26(4)$ \\
\hline Disease severity (GOLD) & \\
\hline Stage II & $18(82 \%)$ \\
\hline Stage III & $4(18 \%)$ \\
\hline GOLD stage & $2.2(0.4)$ \\
\hline MRC score & $2.0(1.1)$ \\
\hline 6MWT (meters) & $515(80)$ \\
\hline Pulmonary function & \\
\hline VC (\% predicted) & $98(18)$ \\
\hline FEV, (liters) & $1.6(0.5)$ \\
\hline FEV 1 ( $\%$ predicted) & $59(11)$ \\
\hline FEV, $/$ FVC & $47(9)$ \\
\hline TLC (\% predicted) & $108(17)$ \\
\hline RV (\% predicted) & $134(34)$ \\
\hline Smoking status & \\
\hline Years smoked & $38(12)$ \\
\hline Pack Years & $30(7)$ \\
\hline Occupation & \\
\hline Retired & $18(82 \%)$ \\
\hline Employed & $3(14 \%)$ \\
\hline On sick-leave & $1(4 \%)$ \\
\hline Data are presented as means (SD) or frequencies (\%). 6MWT: 6 Minute Walk \\
Test; FEV $:$ Forced Expiratory Volume During The First Second; FVC: Forced Vital \\
Capacity; GOLD: Global Initiative For Lung Disease; MRC: Medical Research \\
Council Dyspnea Scale; RV: Residual Volume; TLC: Total Lung Capacity; VC: \\
Vital Capacity
\end{tabular}

Table 1: Characteristics of participants at study entry $(n=22)$.

\begin{tabular}{|c|c|c|c|}
\hline $\begin{array}{l}\text { Center } \\
\text { (Number of physiotherapists) }\end{array}$ & $\begin{array}{l}\text { Umeå } \\
(n=2)\end{array}$ & $\begin{array}{l}\text { Huddinge } \\
(\mathrm{n}=2)\end{array}$ & $\begin{array}{l}\begin{array}{l}\text { Örnsköldsvik } \\
(n=1)\end{array} \\
\text { (n) }\end{array}$ \\
\hline \multicolumn{4}{|c|}{\begin{tabular}{|l|l} 
Experience (in years): & \\
\end{tabular}} \\
\hline As a physiotherapist & $12(10.5-13.5)$ & $13.5(8.75-18.25)$ & 19 \\
\hline With cardio-respiratory patients & $7(5.5-8.5)$ & $6(4-7)$ & 7 \\
\hline Of group training & $11(10.5-11.5)$ & $12(6.5-17.5)$ & 19 \\
\hline Of using elastic bands & $2.5(2.25-2.75)$ & $3(3-3)$ & 3 \\
\hline $\begin{array}{l}\text { No of treated patients in the } \\
\text { study }\end{array}$ & 6 & 12 & 4 \\
\hline \multicolumn{4}{|c|}{$\begin{array}{l}\text { Data is median (interquartile range) if applicable. RT=low load/high repetition } \\
\text { elastic band resistance training }\end{array}$} \\
\hline
\end{tabular}

\section{Attendance}

Twenty-four RT sessions were conducted during the eight-week intervention period. An additional exercise week was offered to enable everyone to reach the minimum goal of 20 exercise sessions. The mean attendance rate was 22.5 out of 24 sessions, i.e. $94 \%$ (91-97) [9,28]. The mean attendance rate without the additional week was $91 \%$ (87-94).

\section{Exercise intensity}

The mean exercise intensity measured as rated muscle fatigue during the intervention was 4.4 out of 5.6 obtained from the baseline 25 repetition maximum test [9], i.e. 79\% (74-83) of the predicted maximum intensity. An increase in resistance, exemplified for the knee extension exercise, of mean $1.7 \mathrm{~kg}(0.9$ to 2.6$)(\mathrm{p}<.001)$, i.e. a $28 \%$ increase in resistance, was seen between the first and last exercise session for all elastic exercises. All participants started at level one in the heel-raise and the step-up exercise. At week eight the median level was $2(1-2.25)(\mathrm{p}<.001)$ and $1(1-2)(\mathrm{p}=.003)$ for single leg heel raise and single leg step-up, respectively.

\section{Adverse events}

Eight participants reported a total number of 28 adverse events distributed over 18 (3.6\%) of the attended exercise sessions. The median rate of sessions in which they experienced adverse event per attended session was 0\% (0-9) for all participants. All adverse events were minor and temporary as judged by the independent pulmonologists as well as by the physiotherapist. There was no disagreement between assessors on any adverse event. The adverse events were musculoskeletal (such as pain or soreness: $64 \%$ ), related to the elastic resistance bands (such as bruising, pain, laceration, swelling: $32 \%$ ), and dizziness (4\%). Seven out of the nine (78\%) adverse events related to the elastic resistance bands was reported during the first seven sessions.

\section{Compliance}

All physiotherapists and all but one patient filled out a questionnaire about the RT regimen. The questions focused on the participants and physiotherapists ability to follow the instructions on the different components of the RT regimen. When rating their own ability to follow the instructions of the RT regimen (when looking at all components together) $96 \%$ (94-96) of participants and 100\% (94-100) physiotherapists, could follow the instructions to a large extent. Regarding compliance to the individual components of the RT regimen, all physiotherapists felt that they could follow the instructions to a large extent with regard to the whole regimen, the eight exercises, the warm-up, the no of repetitions and the ratings of exertion. Fifty percent of the physiotherapists thought that they could follow the instructions to a large extent on the cool-down component of the RT regimen, $71 \%$ regarding the speed of movement component and $88 \%$ regarding the progression component of the RT regimen. There was no difference between the participants and physiotherapists' ratings on any of the different components of the RT regimen regarding the participants' ability to follow the instructions to a large extent (Table 3). In addition, compliance was $96 \%$ and ranged between $88 \%-100 \%$ for the participants and physiotherapists respectively on the components of the RT regimen related to the elastic bands. The results did not differ when compared to the complete case analysis on any of the components of the RT ( $\mathrm{p}=.624-.744)$.

\section{Discussion}

The RT regimen evaluated in this study was feasible for people

\begin{tabular}{|l|l|l|l|}
\hline & $\begin{array}{l}\text { Participants } \\
\text { ability to follow } \\
\text { the instructions } \\
\text { to a large extent } \\
\text { according to } \\
\text { participants } \\
\text { themselves }\end{array}$ & $\begin{array}{l}\text { Participants } \\
\text { ability to follow } \\
\text { the instructions } \\
\text { to a large extent } \\
\text { according to } \\
\text { physiotherapists }\end{array}$ & $\mathbf{P}$ \\
\hline The whole RT regimen & $100 \%$ & $100 \%$ &. $\mathrm{a}$ \\
\hline The warm-up & $96 \%$ & $100 \%$ & $\mathrm{p}=.733$ \\
\hline The eight exercises* & $96 \%$ & $100 \%$ & $\mathrm{p}=.733$ \\
\hline The cool-down & $82 \%$ & $63 \%$ & $\mathrm{p}=.261$ \\
\hline The speed of movement ${ }^{*} \dagger$ & $96 \%$ & $88 \%$ & $\mathrm{p}=.469$ \\
\hline The no. of repetitions* & $96 \%$ & $100 \%$ & $\mathrm{p}=.733$ \\
\hline The ratings of exertion ${ }^{*} \ddagger$ & $91 \%$ & $100 \%$ & $\mathrm{p}=.531$ \\
\hline
\end{tabular}

*Questions related to the elastic bands, fone second in each direction for all but the step-up exercise, $\ddagger$ muscle fatigue and dyspnea. Data is presented in percent. $\mathrm{N} / \mathrm{A}=$ Not applicable, $\mathrm{P}=\mathrm{p}$-value, $\mathrm{RT}=$ low load/high repetition elastic band resistance training a. No statistics are computed because the question is a constant.

Table 3: The percentages of participants who could follow the instructions on the different parts of the RT regimen to a large extent according to a questionnaire $(n=22)$. 
with moderate to severe COPD. There was a high attendance rate, high mean exercise intensity with a significant progression, and a high compliance with the RT regimen for both people with COPD and the physiotherapists performing the RT regimen. Furthermore, there were few adverse events and these were considered minor and temporary.

This study adds to the body of knowledge regarding feasibility of resistance training in general and of elastic band resistance training in specific in people with COPD. The results from this study indicate that an elastic band resistance training regimen is feasible in people with COPD, when performed as low load/high repetition resistance training supervised by physiotherapists in an outpatient setting.

The feasibility of elastic resistance equipment in resistance training for people COPD is of importance since it, in comparison to weight machines, offers a portable and inexpensive alternative that is easy to use and more accessible [29,30].

The attendance rate in the present study was much larger (94\%) than in the elastic band study by O'Shea et al. (56\%) [7]. However, the present RT regimen was performed in group and in an outpatient setting which previously has been shown to have higher completion rates than an individual exercise regimen performed in a home setting [31]. Nevertheless, also in comparison to other studies of resistance training performed in groups of people with COPD the attendance rate was high [3].

We identified a number of adverse events in this study, of which a third (9 out of 28) was related to the elastic bands. A pilot study on one individual with COPD was executed to test the RT regimen in general and the elastic bands in specific [22,23]. However, this may not have been sufficient. The majority of adverse events related to the elastic bands occurred within the first seven exercise sessions. All were considered minor and temporary and were related to how the elastic band was fastened on the exercising limb. Small alterations were made that minimized the rate of adverse events in upcoming sessions. A more comprehensive pilot testing before start of intervention could have identified the initial problems with the elastic bands which may have reduced the number of adverse events related to the equipment.

Compliance to the RT regimen was excellent for both the participants in the RT regimen and the physiotherapists conducting the intervention on the RT in general and on the components of the RT related to the elastic bands in specific. As far as we know this is the first study to evaluate compliance to the different components of a specific exercise regimen in COPD. The enrollment of the physiotherapists as a control on the questionnaire with regard to the participants' ability to follow the instructions of the RT regimen may be considered a bias on the study outcome. The reason for using the physiotherapists as a control was that we anticipated that the participants may have an excessively positive approach to the exercise regimen only for being selected to participate in the study. By comparing the participants opinions with the opinions from the physiotherapists regarding the participants' ability to follow the instructions of the RT regimen we believed that we would get a more balanced and correct result.

Strengths of the present study were the rating of severity of adverse events, the use of raters not involved in the study and the assessment of compliance to the different parts of the RT regimen in both participants and physiotherapists. Other strengths were the comprehensive and standardized description of the RT regimen which facilitates reproducibility and implementation of the intervention. Furthermore, the whole design of the RT regimen focused on clinical applicability.

One limitation in the present study was that no particular instructions were given to the physiotherapists at baseline to note adverse events which may imply a possible underestimation for the occurrence of adverse events. Nevertheless, adverse events were registered and collected from exercise diaries and post-intervention interviews with the physiotherapists and all adverse events that were reported were considered minor and temporary by the independent raters.

Other limitations were that the majority of patients included in the intervention were people with moderate disease and that no participant with very severe COPD was included, as originally intended. This precludes generalization to people with COPD with larger ventilator restraints and muscular dysfunctions. Nevertheless, the results are of importance since people with moderate disease are one of the larger groups in the COPD population [32]. However, it would be of interest to investigate the feasibility of the exercise regimen also in people with were severe disease as adherence to pulmonary rehabilitation has been shown to be lower in those with larger ventilator restraints $[33,34]$. Also, there were some missing data which may have led to bias [26]. However, no differences were seen in comparison with complete case data and there were generally only a few missing observations, which may be considered as a minor disruption $[10,26]$.

The main clinical implication of this study is that this low load/ high repetition elastic band resistance training regimen is feasible for both people with moderate to severe COPD and physiotherapists conducting the intervention. This indicates that elastic bands could be an alternative to weight machines in resistance training for people with COPD. Further studies are needed to evaluate whether the results can be reproduced in a broader range of disease severity and different settings.

\section{Acknowledgments}

We would like to thank the participants for their time and effort participating in this study. We would also like to express our gratitude to the physiotherapists a the University Hospital in Umeå, Karolinska University Hospital in Huddinge, and Örnsköldsvik Hospital in Örnsköldsvik, Sweden, for the supervision and conduction of the intervention. We also thank Lina Schelin for statistical assistance and Monica Edström for assistance with outcome assessment. This study was supported by grants received from the Swedish Research Council (Dnr:522-2009-2694), the Strategic Research Program in Care Sciences (223-654-10), the Medical Faculty of Umeå University (223-3357-09), the Swedish Heart and Lung Foundation (20110214), the Swedish Heart and Lung Association (E160/11) and the County Council of Västerbotten, Sweden.

\section{Conflicts of Interest}

\section{Andre Nyberg has no conflict of interest}

Britta Lindström has no conflict of interest

Karin Wadell has received financial support from the Swedish Research Council (Dnr:522-2009-2694), the Strategic Research Program in Care Sciences (223-654-10), the Medical Faculty of Umeå University (223-3357-09), the Swedish Heart and Lung Foundation (20110214), the Swedish Heart and Lung Association (E160/11), and the County Council of Västerbotten, Sweden. The funders had no role in study design, data collection, analyze, decision to publish, preparation or reporting of the study manuscript.

\section{References}

1. Spruit MA, Singh SJ, Garvey C, ZuWallack R, Nici L, et al. (2013) An official American Thoracic Society/European Respiratory Society statement: key concepts and advances in pulmonary rehabilitation. Am J Respir Crit Care Med 188: 13-64

2. Gosselink R., Troosters T, Decramer M (1996) Peripheral muscle weakness contributes to exercise limitation in COPD. Am J Respir Crit Care Med 153: 976-980.

3. O'Shea SD, Taylor NF, Paratz JD (2009) Progressive resistance exercise improves muscle strength and may improve elements of performance of daily activities for people with COPD: a systematic review 136: 1269-1283. 
Citation: Nyberg A, Lindstrom B, Wadell K (2014) A Cohort Study to Evaluate the Feasibility of Low Load/High Repetition Elastic Band Resistance Training for People with Chronic Obstructive Pulmonary Disease. J Nov Physiother 4: 190. doi:10.4172/2165-7025.1000190

4. Janaudis-Ferreira T, Hill K, Goldstein RS, Robles-Ribeiro P, Beauchamp MK, et al. (2011) Resistance arm training in patients with COPD: A Randomized Controlled Trial 139: 151-158

5. Andersen LL, Andersen $\mathrm{CH}$, Mortensen OS, Poulsen OM, Bjornlund IB, et al. (2010) Muscle activation and perceived loading during rehabilitation exercises: comparison of dumbbells and elastic resistance. Phys Ther 90: 538-549.

6. Jakobsen MD, Sundstrup E, Andersen $\mathrm{CH}$, Bandholm T, Thorborg $\mathrm{K}$, et al. (2012) Muscle activity during knee-extension strengthening exercise performed with elastic tubing and isotonic resistance. Int J Sports Phys Ther 7: 606-616.

7. O'Shea SD, Taylor NF, Paratz JD (2007) A predominantly home-based progressive resistance exercise program increases knee extensor strength in the short-term in people with chronic obstructive pulmonary disease: a randomised controlled trial. Aust J Physiother 53: 229-237.

8. Van't Hul A, Harlaar J, Gosselink R, Hollander P, Postmus P, et al. (2004) Quadriceps muscle endurance in patients with chronic obstructive pulmonary disease. Muscle Nerve 29: 267-274.

9. Nyberg A, Lindstrom B, Wadell K (2012) Assessing the effect of highrepetitive single limb exercises (HRSLE) on exercise capacity and quality of life in patients with chronic obstructive pulmonary disease (COPD): study protocol for randomized controlled trial. Trials13: 114.

10. Vandenbroucke JP, von Elm E, Altman DG, Gotzsche PC, Mulrow CD et al (2007) Strengthening the Reporting of Observational Studies in Epidemiology (STROBE): explanation and elaboration. Epidemiology 18: 805-835.

11. Rabe KF, Hurd S, Anzueto A, Barnes PJ, Buist SA, et al. (2007) Globa strategy for the diagnosis, management, and prevention of chronic obstructive pulmonary disease: GOLD executive summary. Am J Respir Crit Care Med 176: 532-555.

12. American College of Sports Medicine Position Stand (1998). The recommended quantity and quality of exercise for developing and maintaining cardiorespiratory and muscular fitness, and flexibility in healthy adults. Medicine and Science in Sports and Exercise 30: 975-991.

13. Thompson WRG, Pescatello NF, Linda S (2010) ACSM's guidelines for exercise testing and prescription. (8thedn) : Lippincott Williams \& Jenkins Philadelpia, USA

14. American College of Sports Medicine position stand (2009). Progression models in resistance training for healthy adults. Med Sci Sports Exerc 41: 687-708.

15. Bernard S, LeBlanc P, Whittom F, Carrier G, Jobin J, et al. (1998) Peripheral muscle weakness in patients with chronic obstructive pulmonary disease. Am J Respir Crit Care Med 158: 629-634

16. Man WD, Soliman MG, Gearing J, Radford SG, Rafferty GF, et al. (2003) Symptoms and quadriceps fatigability after walking and cycling in chronic obstructive pulmonary disease. Am J Respir Crit Care Med 168: 562-567.

17. Gosker HR, Lencer NH, Franssen FM, van der Vusse GJ, Wouters EF, et al. (2003) Striking similarities in systemic factors contributing to decreased exercise capacity in patients with severe chronic heart failure or COPD. Chest 123: 1416-1424
18. Neptune RR., Zajac FE, Kautz SA (2004) Muscle force redistributes segmental power for body progression during walking. Gait Posture 19: $194-$ 205.

19. Bronstad E, Rognmo O, Tjonna AE, Dedichen HH, Kirkeby-Garstad I, et al. (2012) High intensity knee extensor training restores skeletal muscle function in COPD patients. Eur Respir J 40: 1130-1136.

20. Santiworakul A, Jarungjitaree S, Jalayondeja W, Chantarothorn $S$ Supaibulpipat S (2009) Effect of lower extremity exercise on muscle strength and physical capacity in COPD patients. J Med Assoc Thai 92: 556-563.

21. Escamilla RF, Yamashiro K, Paulos L, Andrews JR (2009) Shoulder muscle activity and function in common shoulder rehabilitation exercises. Sports Med 39: 663-685.

22. Page P, Ellenbecker ST (2003) The scientific and clinical application of elastic resistance. Champaign, IL: Human Kinetics

23. Patterson RM, Stegink Jansen CW, Hogan HA, Nassif MD (2001) Material properties of Thera-Band Tubing. Phys Ther 81: 1437-1445.

24. Borg GA (1982) Psychophysical bases of perceived exertion. Med Sci Sports Exerc 14: 377-381.

25. Littbrand $H$, Rosendahl $E$, Lindel of $N$, Lundin-Olsson L, Gustafson $Y$, et al. (2006) A high-intensity functional weight-bearing exercise program for older people dependent in activities of daily living and living in residential care facilities: evaluation of the applicability with focus on cognitive function. Phys Ther 86: 489-498.

26. Altman DG, Bland JM (2007) Missing data. BMJ 334: 424.

27. Shrive FM, Stuart H, Quan H, Ghali WA (2006) Dealing with missing data in a multi question depression scale: a comparison of imputation methods. BMC Med Res Methodol 6: 57.

28. Jenkins S, Hill K, Cecins NM (2010) State of the art: how to set up a pulmonary rehabilitation program. Respirology 15: 1157-1173.

29. Hostler D, Schwirian CI, Campos G, Toma K, Crill MT, et al. (2001) Skeleta muscle adaptations in elastic resistance-trained young men and women. Eur J Appl Physiol 86: 112-118.

30. Colado JC, Triplett NT (2008) Effects of a short-term resistance program using elastic bands versus weight machines for sedentary middle-aged women. J Strength Cond Res 22: 1441-1448.

31. Hong SY, Hughes S, Prohaska T (2008) Factors affecting exercise attendance and completion in sedentary older adults: a meta-analytic approach. J Phys Act Health 5: 385-397.

32. Jansson SA, Backman H, Stenling A, Lindberg A Rönnmark E, et al. (2013) Health economic costs of COPD in Sweden by disease severity-Has it changed during a ten years period?. Respiratory Medicine: 107:1931-1938.

33. Selzler AM, Simmonds L, Rodgers WM, Wong EY, Stickland MK (2012) Pulmonary rehabilitation in chronic obstructive pulmonary disease: predictors of program completion and success. COPD 9: 538-545.

34. Hayton C, Clark A, Olive S, Browne P, Galey P, et al. (2013) Barriers to pulmonary rehabilitation: characteristics that predict patient attendance and adherence. Respir Med 107: 401-407.

Submit your next manuscript and get advantages of OMICS Group submissions

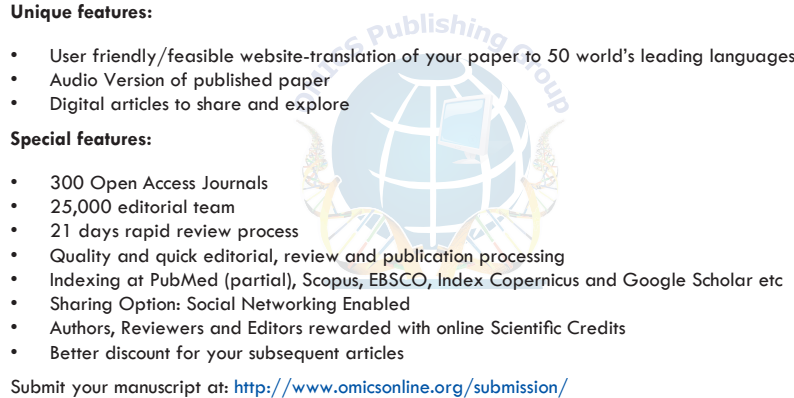

